\title{
Siblings with Ethylmalonic Encephalopathy: Case Report
}

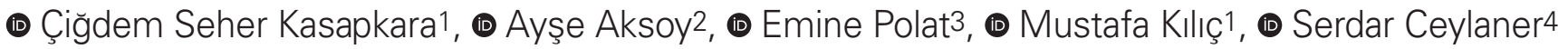 \\ 1 University of Health Sciences, Ankara Dr. Sami Ulus Women and Children's Training and Research Hospital, Clinic of Pediatric Metabolism and \\ Nutrition, Ankara, Turkey \\ 2University of Health Sciences, Ankara Dr. Sami Ulus Women and Children's Training and Research Hospital, Clinic of Pediatric Neurology, Ankara, \\ Turkey \\ 3 University of Health Sciences, Ankara Dr. Sami Ulus Women and Children's Training and Research Hospital, Clinic of Pediatrics, Ankara, Turkey \\ 4 Intergen Genetics Center, Clinic of Medical Genetics, Ankara, Turkey
}

\begin{abstract}
Deficiency of mitochondrial sulfur dioxygenase (ETHE1) causes a rare inborn error of metabolism, ethylmalonic encephalopathy, which is characterized by early-onset encephalopathy, chronic hemorrhagic diarrhea, recurrent petechiae, orthostatic acrocyanosis, defective cytochrome $\mathrm{C}$ oxidase because of hydrogen sulfide accumulation and death in the first years of life. Biochemical hallmarks of the disease are high level of lactate, C4-C5-acylcarnitines in blood and markedly elevated urinary excretion of methylsuccinic and ethylmalonic acids. We report on two siblings who were admitted to a pediatric metabolic unit with acrocyanosis, chronic diarrhea and psychomotor retardation later diagnosed as ethylmalonic encephalopathy. Molecular analyses revealed a homozygous for p.R1630 (c.488 G>A) mutation in ETHE1 gene.

Keywords: Acrocyanosis, developmental delay, ETHE1, ethylmalonic encephalopathy
\end{abstract}

\section{Introduction}

Ethylmalonic encephalopathy (EE) is an autosomal recessive metabolic disease caused by mutations in the mitochondrial sulfur dioxygenase (ETHE1) gene (1). The disease is characterized by an early onset of neurological degeneration, chronic hemorrhagic diarrhea, recurrent petechiae, orthostatic acrocyanosis and death in the first years of life (2-4). Biochemically, these patients have increased urinary excretion of ethylmalonic acid, along with 2-methylsuccinate, butyrylglycine and isovalerylglycine. Along with elevated plasma concentrations of C4 and C5 acylcarnitine species, the patients may experience recurrent or persistent bouts of lactic acidemia and may have cytochrome oxidase deficiency on muscle biopsy. Dysmorphic facial features include the presence of epicanthal folds and a broad nasal bridge. The onset of the disease is during the first months of life and children usually die within the first decade $(5,6)$. We report on siblings with $E E$ who were admitted to the pediatric metabolic unit with acrocyanosis, chronic diarrhea and psychomotor retardation.

\section{Case Report}

A 3 year 9 month old boy was born to consanguineous Turkish parents (first cousins) at term by normal vaginal delivery after an uneventful pregnancy. His growth parameters were as follows: weight $9800 \mathrm{~g}(<3 \mathrm{p})$, length $82 \mathrm{~cm}(<3 \mathrm{p})$, head circumference $48 \mathrm{~cm}(<3 p)$. Their first child, a 6 year and 3 month old female, was born at 39 weeks, with a birth weight of $3600 \mathrm{~g}$. She was firstly admitted to the pediatric nephrology department because of acrocyanosis (Figure 1). Serologic studies were negative for $\mathrm{C}$-reactive protein, serum complement C3, C4, antinuclear antibody, anti-dsDNA and rheumatoid factor. She was admitted to the pediatric metabolic unit with orthostatic acrocyanosis, psychomotor delay and chronic diarrhea. Her growth parameters were as 


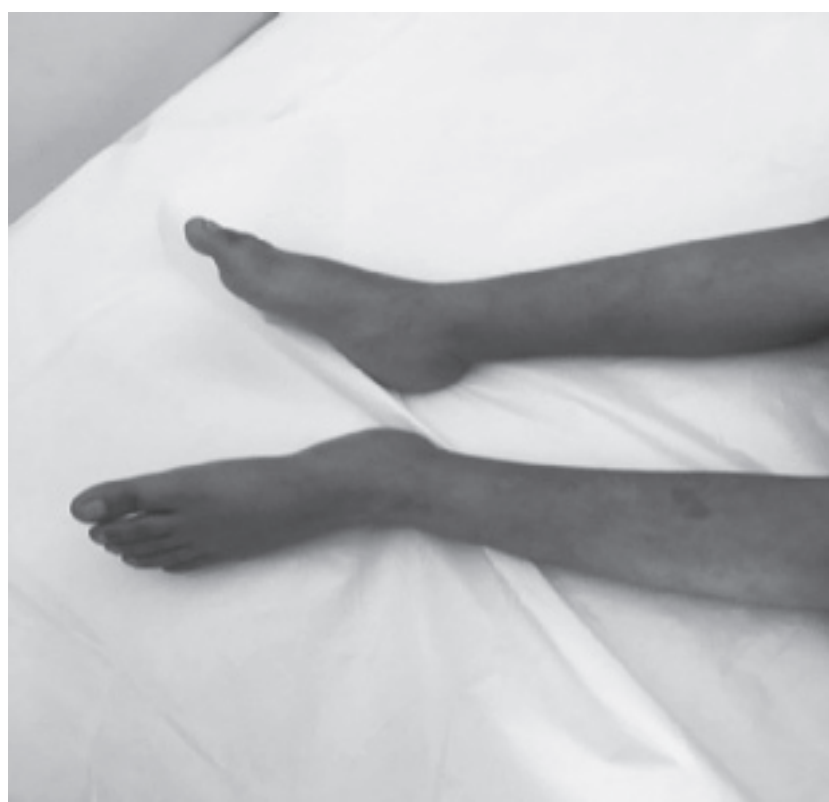

Figure 1. Ortostatic acrocyanosis of the boy with ethylmalonic encephalopathy

follows: weight $10 \mathrm{~kg}(<3 p)$, length $85 \mathrm{~cm}(<3 p)$ and head circumference $48.5 \mathrm{~cm}(<3 p)$. Neurological examination of the siblings revealed axial hypotonia, spasticity of upper and lower extremities, brisk deep tendon reflexes and clonus. This clinical picture led to the suspicion of EE. The elder sister presented all the typical biochemical hallmarks of the disease including elevated lactate and butyrylcarnitine [C4: $2.89 \mu \mathrm{mol} / \mathrm{L}$ normal values (NV: 0.04-1.5)] in blood and elevated urinary excretion of ethylmalonic acid $93.63 \mu \mathrm{mol} / \mathrm{mmol}$ creatinine (NV: 1.714.6), isobutyrylglycine $1.05 \mu \mathrm{mol} / \mathrm{mmol}$ creatinine (NV: 0) and isovalerylglycine $4.9 \mu \mathrm{mol} / \mathrm{mmol}$ creatinine (NV: 0). The second child was clinically similar and had elevated plasma lactate levels on several occasions and urine organic acids consistently showed elevations of ethylmalonic acid [83 $\mu \mathrm{mol} / \mathrm{mmol}$ creatinine (NV: 1.7-14.6)], methylsuccinate, isobutyrylglycine $2.62 \mu \mathrm{mol} / \mathrm{mmol}$ creatinine (NV: 0) and isovalerylglycine $11.95 \mu \mathrm{mol} / \mathrm{mmol}$ creatinine (NV: 0 ). Plasma acylcarnitine analyses showed elevations of short chain species of butyrylcarnitine and isovalerylcarnitine. A peripheral blood sample was collected from the patient after we obtained informed consent. The ETHE1 gene was sequenced and they were both shown to be homozygous for the p.R1630 (c.488G>A) mutation while both of the parents were heterozygous for the same mutation. The p.R1630 (c.488G>A) mutation has been described previously in EE. On follow-up, combined treatment with oral metronidazole $30 \mathrm{mg} / \mathrm{kg} /$ day, $\mathrm{N}$-acetylcysteine $100 \mathrm{mg} /$ $\mathrm{kg} /$ day, coenzyme $0105 \mathrm{mg} / \mathrm{kg} /$ day and riboflavin $100 \mathrm{mg} /$ day were administered with clinical improvement (mild neurological improvement, partial control of diarrhea and disappearance of acrocyanosis).

\section{Discussion}

EE (EE; OMIM No. 602473) is a fatal infantile disease caused by an accumulation of sulfide, $\mathrm{H}_{2} \mathrm{~S}$, a mitochondrial poison produced exogenously by the anaerobic enterobacterial flora and synthesized endogenously in various mammalian tissues (5). Failure to detoxify sulfide is due to the absence or malfunctioning of a mitochondrial sulfur dioxygenase, encoded by the ETHE1 gene, which is mutated in EE, and characterized by ethylmalonic and methylsuccinic aciduria and lactic acidemia associated with neurodevelopmental delay and regression, pyramidal and extrapyramidal signs, vascular lesions determining episodes of acrocyanosis, recurrent petechiaea and chronic diarrhea (6-8).Our patients had acrocyanosis, chronic diarrhea and psychomotor delay. Acrocyanosis appears as a bluish discoloration of the skin and mucous membrane because of diminished oxyhemoglobin. It is caused by chronic vasospasm of small cutaneous arteries and arterioles along with compensatory dilatation in the capillaries. It can be both primary and secondary to psychiatric, neurologic, autoimmune, infective and metabolic causes. Orthostatic acrocyanosis is one of the principal signs of EE. Inherited metabolic disorders causing acrocyanosis also include fucosidosis, hyperoxaluria Type I, congenital disorder of glycosylation and mitochondrial disorders with onset in early infancy and multisystem involvement. Persistent anorexia, feeding difficulties, chronic vomiting, failure to thrive, frequent infections, generalized hypotonia and neurological findings in association with chronic diarrhea occur in a wide variety of inborn errors of metabolism. Mitochondrial Neurogastrointestinal Encephalopathy (MNGIE) syndrome, menkes disease, congenital disorder of glycosylation, hartnup disease and disorders of cobalamin and folate metabolism and transport are examples of metabolic disorders which also give rise to chronic diarrhea and neurological findings (2-4). High levels of ethylmalonic acid in the urine may also be observed inshort-chain acyl-CoA dehydrogenase deficiency and glutaric acidemia Type II, but none of these has been associated with the major clinical features of EE. Many patients have pyramidal or extrapyramidal signs, hypotonia, microcephaly, failure to thrive, seizures and episodes of coma. Combined treatment with oral metronidazole, N-acetylcysteine, riboflavinand coenzyme $\mathrm{Q} 10$ resulted in marked neurological improvement, disappearance of diarrhea and acrocyanosis in our patients $(9,10)$. In conclusion, the possibility of EE should be investigated and examined in patients presenting with chronic diarrhea, early onset of neurological degeneration, recurrent petechiae and orthostatic acrocyanosis.

\section{Ethics}

Informed Consent: The parents of the children reported here have consented to these studies.

Peer-review: External and internal peer-reviewed. 


\section{Authorship Contributions}

Surgical and Medical Practices: Ç.S.K., S.C., Concept: C..S.K., Design: Ç.S.K., S.C., Data Collection or Processing: C..S.K., S.C., Analysis or Interpretation: Ç.S.K., S.C., Literature Search: Ç.S.K., Writing: C..S.K., A.A., E.P., M.K., S.C.

Conflict of Interest: No conflict of interest was declared by the authors.

Financial Disclosure: The authors declared that this study received no financial support.

\section{References}

1. Bijarnia-Mahay S, Gupta D, Shigematsu Y, et al. Ethylmalonic Encephalopathy in an Indian Boy. Indian Pediatr 2016;53:9146.

2. Yiş U, Polat I, Karakaya P, Ayanoğlu M, Hiz AS. Importance of acrocyanosis indelayed walking. J Pediatr Neurosci 2015;10:80-1.

3. Henriques BJ, Lucas TG, Rodrigues JV, et al. Ethylmalonic encephalopathy ETHE1 R163W/R1630mutations alterprotein stability and redox properties of the iron centre. PLoS One 2014;9:e107157.

4. Tiranti $\mathrm{V}$, Zeviani $\mathrm{M}$. Altered sulfide $(\mathrm{H}(2) \mathrm{S})$ metabolism in ethylmalonicencephalopathy. Cold Spring Harb Perspect Biol 2013;5:a011437.
5. Pavlou E, Augoustides-Savvopoulou P, Gregersen N, et al.An infant with ethylmalonic encephalopathymasquerading as a hematologic disorder. J Child Neurol 2013;28:668-71.

6. Dweikat I, Naser E, Damsah N, Libdeh BA, Bakri I. Ethylmalonic encephalopathyassociated with crescentic glomerulonephritis. Metab Brain Dis 2012;27:613-6.

7. Giordano C, Viscomi C, Orlandi M, et al. Morphologic evidence of diffusevascular damage in human and in the experimental model of ethylmalonicencephalopathy. $J$ Inherit Metab Dis 2012;35:451-8.

8. Walsh DJ, Sills ES, Lambert DM, et al.Novel ETHE1 mutation in a carrier couple having prior offspring affected with ethylmalonicencephalopathy: Genetic analysis, clinical management and reproductive outcome.Mol Med Rep 2010;3:223-6.

9. Palmfeldt J, Vang S, Stenbroen V, et al. Proteomics reveals that redoxregulation is disrupted in patients with ethylmalonic encephalopathy.J ProteomeRes 2011;10:238996.

10. Di Meo I, Fagiolari G, Prelle A, et al. Chronicexposure to sulfide causes accelerated degradation of cytochrome $\mathrm{c}$ oxidase inethylmalonic encephalopathy. Antioxid Redox Signal 2011;15:353-62. 